\title{
In-Datacenter Performance Analysis of a Tensor Processing Unit
}

Norman P. Jouppi, Cliff Young, Nishant Patil, David Patterson, Gaurav Agrawal, Raminder Bajwa, Sarah Bates, Suresh Bhatia, Nan Boden, Al Borchers, Rick Boyle, Pierre-luc Cantin, Clifford Chao, Chris Clark, Jeremy Coriell, Mike Daley, Matt Dau, Jeffrey Dean, Ben Gelb, Tara Vazir Ghaemmaghami, Rajendra Gottipati, William Gulland, Robert Hagmann, C. Richard Ho, Doug Hogberg, John Hu, Robert Hundt, Dan Hurt, Julian Ibarz, Aaron Jaffey, Alek Jaworski, Alexander Kaplan, Harshit Khaitan, Daniel Killebrew, Andy Koch, Naveen Kumar, Steve Lacy, James Laudon, James Law, Diemthu Le, Chris Leary, Zhuyuan Liu, Kyle Lucke, Alan Lundin, Gordon MacKean, Adriana Maggiore, Maire Mahony, Kieran Miller, Rahul Nagarajan, Ravi Narayanaswami, Ray Ni, Kathy Nix, Thomas Norrie, Mark Omernick, Narayana Penukonda, Andy Phelps, Jonathan Ross, Matt Ross, Amir Salek, Emad Samadiani, Chris Severn, Gregory Sizikov, Matthew Snelham, Jed Souter, Dan Steinberg, Andy Swing, Mercedes Tan, Gregory Thorson, Bo Tian, Horia Toma, Erick Tuttle, Vijay Vasudevan, Richard Walter, Walter Wang, Eric Wilcox, and Doe Hyun Yoon

Google, Inc., Mountain View, CA USA jouppi@google.com

\begin{abstract}
Many architects believe that major improvements in cost-energyperformance must now come from domain-specific hardware. This paper evaluates a custom ASIC-called a Tensor Processing Unit (TPU) - deployed in datacenters since 2015 that accelerates the inference phase of neural networks $(\mathrm{NN})$. The heart of the TPU is a 65,536 8-bit MAC matrix multiply unit that offers a peak throughput of 92 TeraOps/second (TOPS) and a large (28 MiB) software-managed on-chip memory. The TPU's deterministic execution model is a better match to the 99th-percentile responsetime requirement of our NN applications than are the time-varying optimizations of CPUs and GPUs that help average throughput more than guaranteed latency. The lack of such features helps explain why, despite having myriad MACs and a big memory, the TPU is relatively small and low power. We compare the TPU to a server-class Intel Haswell CPU and an Nvidia K80 GPU, which are contemporaries deployed in the same datacenters. Our workload, written in the high-level TensorFlow framework, uses production NN applications (MLPs, CNNs, and LSTMs) that represent $95 \%$ of our datacenters' NN inference demand. Despite low utilization for some applications, the TPU is on average about $15 \mathrm{X}-30 \mathrm{X}$ faster than its contemporary GPU or CPU, with TOPS/Watt about $30 \mathrm{X}-80 \mathrm{X}$ higher. Moreover, using the GPU's GDDR5 memory in the TPU would triple achieved TOPS and raise TOPS/Watt to nearly $70 \mathrm{X}$ the GPU and $200 \mathrm{X}$ the CPU.
\end{abstract}

\section{CCS CONCEPTS}

- Computer systems organization $\rightarrow$ Neural Networks

Permission to make digital or hard copies of part or all of this work for personal or classroom use is granted without fee provided that copies are not made or distributed for profit or commercial advantage and that copies bear this notice and the full citation on the first page. Copyrights for third-party components of this work must be honored. For all other uses, contact the Owner/Author. ISCA '17, June 24-28, 2017, Toronto, ON, Canada (C) 2017 Copyright is held by the owner/author(s). ACM ISBN 978-1-4503-4892-8/17/06.

https://doi.org/10.1145/3079856.3080246

\section{KEYWORDS}

DNN, MLP, CNN, RNN, LSTM, neural network, deep learning, domain-specific architecture, accelerator, TensorFlow, TPU, GPU

\section{ACM Reference format:}

Norman P. Jouppi, Cliff Young, Nishant Patil, David Patterson, et al., Google, Inc., Mountain View, CA USA 2017. In-Datacenter Performance Analysis of a Tensor Processing Unit. In Proceedings of ISCA '17, Toronto, ON, Canada, June 24-28, 2017, 12 pages.

https://doi.org/10.1145/3079856.3080246

\section{INTRODUCTION TO NEURAL NETWORKS}

The synergy between the large data sets in the cloud and the numerous computers that power it has enabled a renaissance in machine learning. In particular, deep neural networks (DNNs) have led to breakthroughs such as reducing word error rates in speech recognition by $30 \%$ over traditional approaches, which was the biggest gain in 20 years [17]; cutting the error rate in an image recognition competition since 2011 from $26 \%$ to $3.5 \%$ [30, $56,22]$; and beating a human champion at Go [53].

Neural networks $(\mathrm{NN})$ target brain-like functionality and are based on a simple artificial neuron: a nonlinear function (such as $\max (0$, value $))$ of a weighted sum of the inputs. These pseudo neurons are collected into layers, with the outputs of one layer becoming the inputs of the next in the sequence. The "deep" part of DNN comes from going beyond a few layers, as the large data sets in the cloud allowed more accurate models to be built by using extra and larger layers to capture higher levels of patterns or concepts, and GPUs provided enough computing to develop them.

The two phases of NN are called training (or learning) and inference (or prediction), and they refer to development versus production. The developer chooses the number of layers and the type of $\mathrm{NN}$, and training determines the weights. Virtually all training today is in floating point, which is one reason GPUs have been so popular. A step called quantization transforms floatingpoint numbers into narrow integers-often just 8 bits-which are usually good enough for inference. Eight-bit integer multiplies can be $6 X$ less energy and 6X less area than IEEE 754 16-bit 
floating-point multiplies, and the advantage for integer addition is $13 \mathrm{X}$ in energy and $38 \mathrm{X}$ in area [15].

Three kinds of NNs are popular today:

1. Multi-Layer Perceptrons (MLP): Each new layer is a set of nonlinear functions of a weighted sum of all outputs (fully connected) from the prior one.

2. Convolutional Neural Networks (CNN): Each layer is a set of nonlinear functions of weighted sums at different coordinates of spatially nearby subsets of outputs from the prior layer, which allows the weights to be reused.

3. Recurrent Neural Networks (RNN): Each subsequent layer is a collection of nonlinear functions of weighted sums of outputs and the previous state. The most popular RNN is Long Short-Term Memory (LSTM). The art of the LSTM is in deciding what to forget and what to pass on as state to the next layer. The weights are reused across time steps.

Table 1 shows two examples of each of the three types of NNs-which represent $95 \%$ of NN inference workload in our datacenters - that we use as benchmarks. Typically written in TensorFlow [1], they are surprisingly short: just 100 to 1500 lines of code. Our benchmarks are small pieces of larger applications that run on the host server, which can be thousands to millions of lines of $\mathrm{C}++$ code. The applications are typically user facing, which leads to rigid response-time limits.

Each model needs between 5M and 100M weights (9th column of Table 1), which can take a lot of time and energy to access. To amortize the access costs, the same weights are reused across a batch of independent examples during inference or training, which improves performance.

This paper describes and measures the Tensor Processing Unit (TPU) and compares its performance and power for inference to its contemporary CPUs and GPUs. Here is a preview of the highlights:

- Inference apps usually emphasize response-time over throughput since they are often user facing.

- As a result of latency limits, the K80 GPU is just a little faster for inference than the Haswell CPU, despite it having much higher peak performance and memory bandwidth.

- While most architects are accelerating CNNs, they are just $5 \%$ of our datacenter workload.

- The TPU is about $15 \mathrm{X}-30 \mathrm{X}$ faster at inference than the $\mathrm{K} 80$ GPU and the Haswell CPU.
- $\quad$ Four of the six NN apps are memory bound; if the TPU were revised to have the same memory as the K80 GPU, it would be about $30 \mathrm{X}-50 \mathrm{X}$ faster than the GPU and CPU.

- Despite having a much smaller and lower power chip, the TPU has 25 times as many MACs and 3.5 times as much onchip memory as the K80 GPU.

- The performance/Watt of the TPU is $30 \mathrm{X}-80 \mathrm{X}$ that of its contemporary CPUs and GPUs; a revised TPU with K80 memory would be $70 \mathrm{X}-200 \mathrm{X}$ better.

\section{TPU ORIGIN, ARCHITECTURE, IMPLEMENTATION, AND SOFTWARE}

Starting as early as 2006, we discussed deploying GPUs, FPGAs, or custom ASICs in our datacenters. We concluded that the few applications that could run on special hardware could be done virtually for free using the excess capacity of our large datacenters, and it's hard to improve on free. That changed in 2013 when a projection showed people searching by voice for three minutes a day using speech recognition DNNs would double our datacenters' computation demands, which would be very expensive using conventional CPUs. Thus, we started a highpriority project to produce a custom ASIC quickly for inference (and bought off-the-shelf GPUs for training). The goal was to improve cost-performance by $10 \mathrm{X}$ over GPUs. Given this mandate, in just 15 months the TPU was designed, verified [55], built, and deployed in datacenters. (Space limits the amount and the level of detail on the TPU in this paper; see [46], [47], [48], [49], [57], and [60] for more.)

Rather than be tightly integrated with a CPU, to reduce the chances of delaying deployment, the TPU was designed to be a coprocessor on the PCIe I/O bus, allowing it to plug into existing servers just as a GPU does. Moreover, to simplify hardware design and debugging, the host server sends TPU instructions for it to execute rather than the TPU fetching them itself. Hence, the TPU is closer in spirit to an FPU (floating-point unit) coprocessor than it is to a GPU.

The goal was to run whole inference models in the TPU to reduce interactions with the host $\mathrm{CPU}$ and to be flexible enough to match the NN needs of 2015 and beyond, instead of just what was required for 2013 NNs. Figure 1 shows the block diagram of the TPU.

The TPU instructions are sent from the host over the PCIe Gen 3 x 16 bus into an instruction buffer. The internal blocks are typically connected together by 256-byte-wide paths. Starting in

\begin{tabular}{|c|c|c|c|c|c|c|c|c|c|c|c|}
\hline \multirow{2}{*}{ Name } & \multirow{2}{*}{$L O C$} & \multicolumn{5}{|c|}{ Layers } & \multirow{2}{*}{$\begin{array}{l}\text { Nonlinear } \\
\text { function }\end{array}$} & \multirow{2}{*}{ Weights } & \multirow{2}{*}{$\begin{array}{c}\text { TPU Ops / } \\
\text { Weight Byte }\end{array}$} & \multirow{2}{*}{$\begin{array}{c}\text { TPU Batch } \\
\text { Size }\end{array}$} & \multirow{2}{*}{$\begin{array}{c}\text { \% of Deployed TPUs } \\
\text { in July } 2016\end{array}$} \\
\hline & & $F C$ & Conv & Vector & Pool & Total & & & & & \\
\hline MLP0 & 100 & 5 & & & & 5 & ReLU & $20 \mathrm{M}$ & 200 & 200 & \multirow{2}{*}{$61 \%$} \\
\hline MLP1 & 1000 & 4 & & & & 4 & ReLU & $5 \mathrm{M}$ & 168 & 168 & \\
\hline LSTM0 & 1000 & 24 & & 34 & & 58 & sigmoid, tanh & $52 \mathrm{M}$ & 64 & 64 & \multirow{2}{*}{$29 \%$} \\
\hline LSTM1 & 1500 & 37 & & 19 & & 56 & sigmoid, tanh & $34 \mathrm{M}$ & 96 & 96 & \\
\hline CNNO & 1000 & & 16 & & & 16 & ReLU & $8 \mathrm{M}$ & 2888 & 8 & \multirow{2}{*}{$5 \%$} \\
\hline CNN1 & 1000 & 4 & 72 & & 13 & 89 & ReLU & $100 \mathrm{M}$ & 1750 & 32 & \\
\hline
\end{tabular}

Table 1. Six NN applications ( 2 per NN type) that represent $95 \%$ of the TPU's workload. The columns are the NN name; lines of code; types and number of layers in the NN (FC is fully connected, Conv is convolution, Vector is self-explanatory, Pool is pooling, which does nonlinear downsizing on the TPU; nonlinear function; number of weights; TPU operations per byte (operational intensity in Figure 5); batch size; and TPU application popularity in July 2016. One DNN is RankBrain [14]; one LSTM is a subset of GNM Translate [59]; one CNN is Inception; and the other CNN is DeepMind AlphaGo [53, 27]. 
the upper-right corner, the Matrix Multiply Unit is the heart of the TPU. It contains 256x256 MACs that can perform 8-bit multiplyand-adds on signed or unsigned integers. The 16-bit products are collected in the $4 \mathrm{MiB}$ of 32-bit Accumulators below the matrix unit. The $4 \mathrm{MiB}$ holds 4096, 256-element, 32-bit accumulators. The matrix unit produces one 256-element partial sum per clock cycle. We picked 4096 by first noting that the operations per byte needed to reach peak performance (roofline knee in Section 4) is $\sim 1350$, so we rounded that up to 2048 and then duplicated it so that the compiler could use double buffering while running at peak performance.

When using a mix of 8-bit weights and 16-bit activations (or vice versa), the Matrix Unit computes at half-speed, and it computes at a quarter-speed when both are 16 bits. It reads and writes 256 values per clock cycle and can perform either a matrix multiply or a convolution. The matrix unit holds one $64 \mathrm{KiB}$ tile of weights plus one for double buffering (to hide the 256 cycles it takes to shift a tile in). This unit is designed for dense matrices. Sparse architectural support was omitted for time-to-deployment reasons. The weights for the matrix unit are staged through an onchip Weight FIFO that reads from an off-chip $8 \mathrm{GiB}$ DRAM called Weight Memory (for inference, weights are read-only; 8 GiB supports many simultaneously active models). The weight FIFO is four tiles deep. The intermediate results are held in the 24 MiB on-chip Unified Buffer, which can serve as inputs to the Matrix Unit. A programmable DMA controller transfers data to or from CPU Host memory and the Unified Buffer.

Figure 2 shows the floor plan of the TPU die. The $24 \mathrm{MiB}$ Unified Buffer is almost a third of the die and the Matrix Multiply Unit is a quarter, so the datapath is nearly two-thirds of the die. The $24 \mathrm{MiB}$ size was picked in part to match the pitch of the Matrix Unit on the die and, given the short development schedule, in part to simplify the compiler (see Section 7). Control is just $2 \%$. Figure 3 shows the TPU on its printed circuit card, which inserts into existing servers like an SATA disk.

As instructions are sent over the relatively slow PCIe bus, TPU instructions follow the CISC tradition, including a repeat field. The average clock cycles per instruction (CPI) of these CISC instructions is typically 10 to 20 . It has about a dozen instructions overall, but these five are the key ones:

1. Read_Host_Memory reads data from the CPU host memory into the Unified Buffer (UB).

2. Read_Weights reads weights from Weight Memory into the Weight FIFO as input to the Matrix Unit.

3. MatrixMultiply/Convolve causes the Matrix Unit to perform a matrix multiply or a convolution from the Unified Buffer into the Accumulators. A matrix operation takes a variable-sized $\mathrm{B} * 256$ input, multiplies it by a $256 \times 256$ constant weight input, and produces a $\mathrm{B} * 256$ output, taking B pipelined cycles to complete.

4. Activate performs the nonlinear function of the artificial neuron, with options for ReLU, Sigmoid, and so on. Its inputs are the Accumulators, and its output is the Unified Buffer. It can also perform the pooling operations needed for convolutions using the dedicated hardware on the die, as it is connected to nonlinear function logic.

5. Write_Host_Memory writes data from the Unified Buffer into the CPU host memory.

The other instructions are alternate host memory read/write, set configuration, two versions of synchronization, interrupt host,

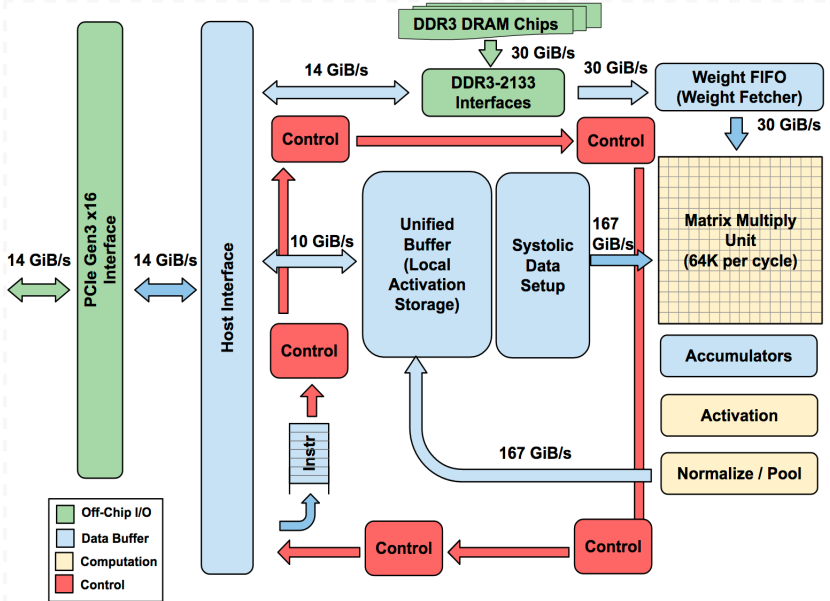

Figure 1. TPU Block Diagram. The main computation is the yellow Matrix Multiply unit. Its inputs are the blue Weight FIFO and the blue Unified Buffer and its output is the blue Accumulators. The yellow Activation Unit performs the nonlinear functions on the Accumulators, which go to the Unified Buffer.

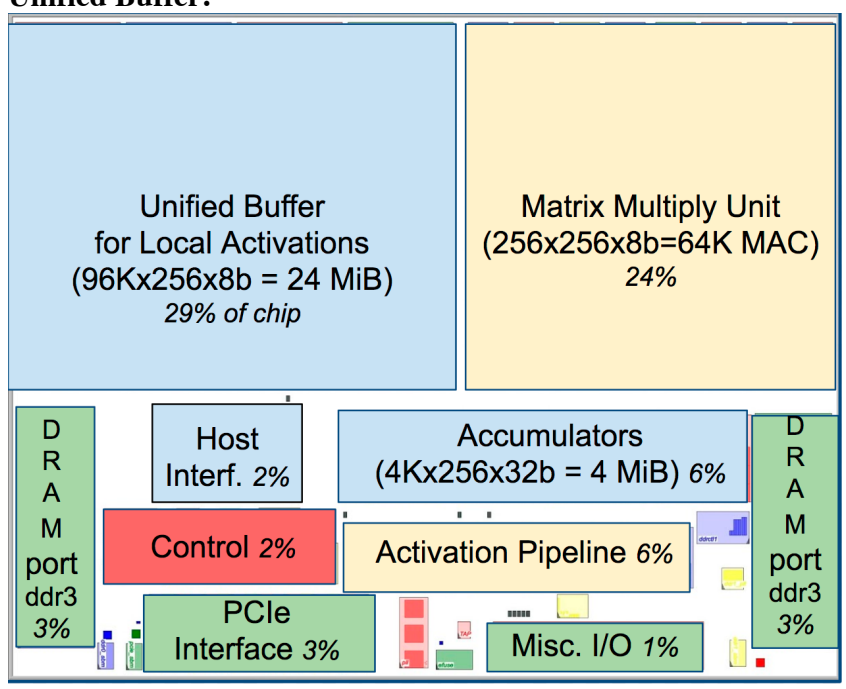

Figure 2. Floorplan of TPU die. The shading follows Figure 1. The light (blue) datapath is $67 \%$, the medium (green) $\mathrm{I} / \mathrm{O}$ is $10 \%$, and the dark (red) control is just $2 \%$ of the die. Control is much larger (and much harder to design) in a CPU or GPU.

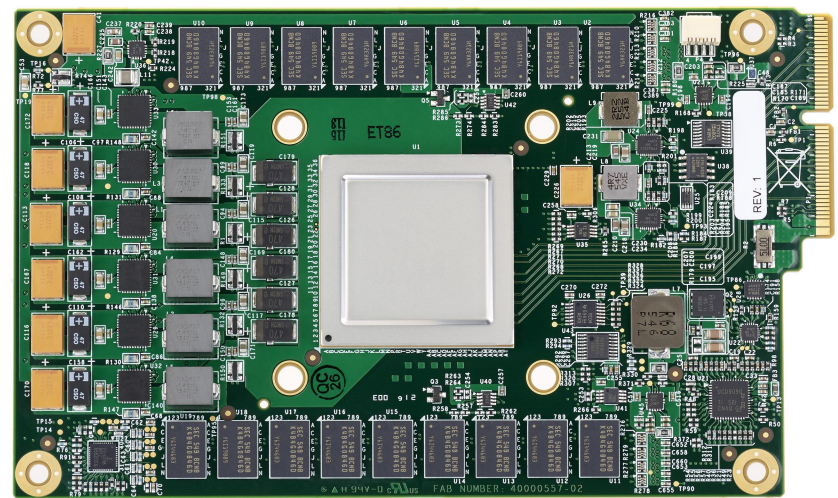

Figure 3. TPU Printed Circuit Board. It can be inserted into the slot for a SATA disk in a server. 
debug-tag, nop, and halt. The CISC MatrixMultiply instruction is 12 bytes, of which 3 are Unified Buffer address; 2 are accumulator address; 4 are length (2 dimensions for convolutions); and the rest are opcode and flags.

The philosophy of the TPU microarchitecture is to keep the matrix unit busy. It uses a 4-stage pipeline for these CISC instructions, where each instruction executes in a separate stage. The plan was to hide the execution of the other instructions by overlapping their execution with the MatrixMultiply instruction. Toward that end, the Read Weights instruction follows the decoupled-access/execute philosophy [54], in that it can complete after sending its address but before the weights are fetched from Weight Memory. The matrix unit will stall if the input activation or weight data is not ready.

We don't have clean pipeline overlap diagrams, because our CISC instructions can occupy a station for 1000 s of clock cycles, unlike the traditional RISC pipeline with 1 per stage. Situations occur when the activations for one network layer must complete before the matrix multiplications of the next layer can begin; we see a RAW pipeline stall where the matrix unit waits for explicit synchronization before safely reading from the Unified Buffer.

As reading a large SRAM uses much more power than arithmetic, the matrix unit uses systolic execution to save energy by reducing reads and writes of the Unified Buffer [31, 44, 40]. It relies on data from different directions arriving at cells in an array at regular intervals where they are combined. Figure 4 shows that data flows in from the left, and the weights are loaded from the top. A given 256-element multiply-accumulate operation moves through the matrix as a diagonal wavefront. The weights are preloaded, and take effect with the advancing wave alongside the first data of a new block. Control and data are pipelined to give the illusion that the 256 inputs are read at once, and that they instantly update one location of each of 256 accumulators. From a correctness perspective, software is unaware of the systolic nature of the matrix unit, but for performance, it does worry about the latency of the unit.

The TPU software stack had to be compatible with those developed for CPUs and GPUs so that applications could be ported quickly to the TPU. The portion of the application run on the TPU is typically written in TensorFlow and is compiled into an API that can run on GPUs or TPUs [33]. Like GPUs, the TPU stack is split into a User Space Driver and a Kernel Driver. The Kernel Driver is lightweight and handles only memory management and interrupts. It is designed for long-term stability.

The User Space driver changes frequently. It sets up and controls TPU execution, reformats data into TPU order, translates
API calls into TPU instructions, and turns them into an application binary. The User Space driver compiles a model the first time it is evaluated, caching the program image and writing the weight image into the TPU's weight memory; the second and following evaluations run at full speed. The TPU runs most models completely from inputs to outputs, maximizing the ratio of TPU compute time to $\mathrm{I} / \mathrm{O}$ time. Computation is often done one layer at a time, with overlapped execution allowing the matrix multiply unit to hide most non-critical-path operations.

\section{CPU, GPU, AND TPU PLATFORMS}

The six production applications in Table 1 are our workload for this paper. As mentioned above, these six are representative of $95 \%$ of TPU use in our datacenters. Ironically, deploying and measuring popular small DNNs like AlexNet or VGG is difficult on production machines. However, one of our CNNs derives from Inception V2, which is widely used.

The benchmark platforms are server-class computers that were available in 2015 when the TPUs were deployed. This restriction meant that they must include at least SECDED protection of internal SRAM as well as external DRAM memory like the TPU, which excludes some choices such as the Nvidia Maxwell GPU. For our company to purchase and deploy them, they also had to be sensibly configured machines, and not awkward artifacts assembled solely to win benchmarks.

Table 2 lists our choices. The traditional CPU server is represented by an 18-core, dual-socket Haswell processor from

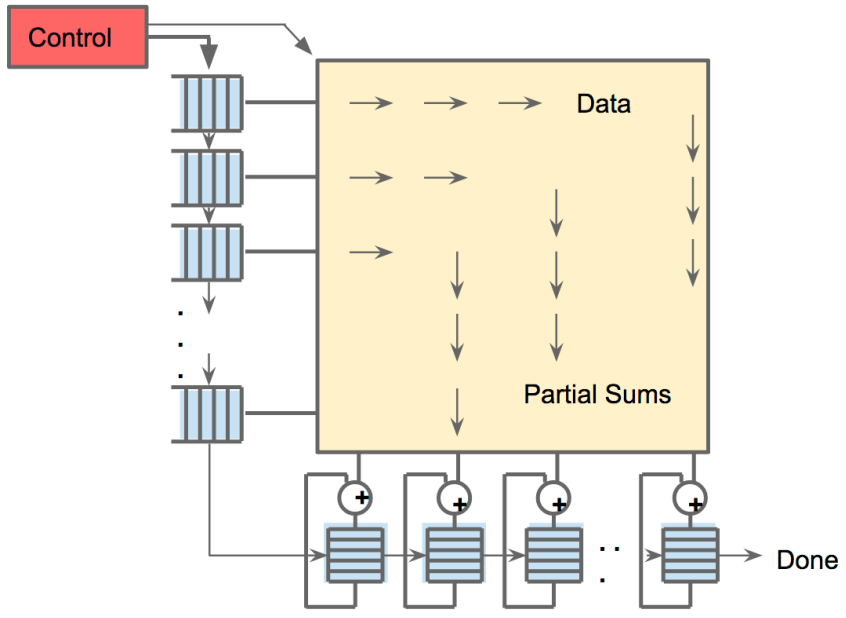

Figure 4. Systolic data flow of the Matrix Multiply Unit.

\begin{tabular}{|c|c|c|c|c|c|c|c|c|c|c|c|c|c|c|c|}
\hline \multirow{3}{*}{ Model } & \multicolumn{10}{|c|}{ Die } & \multicolumn{5}{|c|}{ Benchmarked Servers } \\
\hline & \multirow{2}{*}{$\mathrm{mm}^{2}$} & \multirow{2}{*}{$n m$} & \multirow{2}{*}{$M H z$} & \multirow{2}{*}{$T D P$} & \multicolumn{2}{|c|}{ Measured } & \multicolumn{2}{|c|}{$T O P S / s$} & \multirow{2}{*}{$G B / s$} & \multirow{2}{*}{$\begin{array}{l}\text { On-Chip } \\
\text { Memory }\end{array}$} & \multirow{2}{*}{ Dies } & \multirow{2}{*}{ DRAM Size } & \multirow{2}{*}{$T D P$} & \multicolumn{2}{|c|}{ Measured } \\
\hline & & & & & Idle & Busy & $8 b$ & FP & & & & & & Idle & Busy \\
\hline $\begin{array}{l}\text { Haswell } \\
\text { E5-2699 v3 }\end{array}$ & 662 & 22 & 2300 & $145 \mathrm{~W}$ & $41 \mathrm{~W}$ & $145 \mathrm{~W}$ & 2.6 & 1.3 & 51 & $51 \mathrm{MiB}$ & 2 & 25 & $504 W$ & $159 W$ & $455 \mathrm{~W}$ \\
\hline $\begin{array}{l}\text { NVIDIA K80 } \\
(2 \text { dies/card) }\end{array}$ & 561 & 28 & 560 & $150 \mathrm{~W}$ & $25 \mathrm{~W}$ & $98 \mathrm{~W}$ & -- & 2.8 & 160 & $8 \mathrm{MiB}$ & 8 & $\begin{array}{c}256 \mathrm{GiB} \text { (host) } \\
+12 \mathrm{GiB} \times 8\end{array}$ & $1838 \mathrm{~W}$ & $357 \mathrm{~W}$ & $991 \mathrm{~W}$ \\
\hline TPU & $<331 *$ & 28 & 700 & $75 \mathrm{~W}$ & $28 \mathrm{~W}$ & $40 \mathrm{~W}$ & 92 & -- & 34 & $28 \mathrm{MiB}$ & 4 & $\begin{array}{c}256 \mathrm{GiB} \text { (host) } \\
+8 \mathrm{GiB} \times 4\end{array}$ & $861 W$ & $290 \mathrm{~W}$ & $384 W$ \\
\hline
\end{tabular}

Table 2. Benchmarked servers use Haswell CPUs, K80 GPUs, and TPUs. Haswell has 18 cores, and the K80 has 13 SMX processors. Figure 10 shows measured power. The low-power TPU allows for better rack-level density than the high-power GPU. The 8 GiB DRAM per TPU is Weight Memory. GPU Boost mode is not used (see Section 8). SECDED and no Boost mode reduce K80 bandwidth from its advertised 240 to $160 \mathrm{~GB} / \mathrm{s}$. No Boost mode and single die vs. dual die performance reduces advertised K80 peak TOPS/s from 8.7 to 2.8. (*The TPU die is less than half the Haswell die size.) 
Intel. This platform is also the host server for GPUs or TPUs. Haswell was fabbed in an Intel $22 \mathrm{~nm}$ process. Both the CPU and GPU are very large dies: about $600 \mathrm{~mm}^{2}$ !

The $2.3 \mathrm{GHz}$ CPU clock rate doesn't include Turbo mode because it seldom occurs in our datacenters for NN apps. Haswell has different clock rates depending on whether programs use AVX instructions, which our NN apps often use. The higher clock rate of Turbo mode (for programs that avoid AVX) occurs when they don't use all their cores. Thus, another reason Turbo mode is rare in our datacenters is that our apps typically do use all the cores, plus they can run other datacenter jobs to fill any idle cores.

The GPU accelerator is the Nvidia K80. Each K80 card contains two dies and offers SECDED on internal memory and DRAM. Nvidia states that the "K80 Accelerator dramatically lowers datacenter cost by delivering application performance with fewer, more powerful servers" [38]. NN researchers frequently used K80s in 2015, and they were chosen for new cloud-based GPU offerings as recently as September 2016 [7]. Up to eight K80 dies can be installed in four cards on this server, which is the configuration we benchmark.

As the number of dies per server varies between 2 to 8 , we usually show results normalized per die (Figures 5-8, Figures 1011, and Tables 3, 5, and 7), but we occasionally show whole systems (Figure 9). We hope this distinction is clear.

\section{PERFORMANCE: ROOFLINES, RESPONSE- TIME, AND THROUGHPUT}

To illustrate the performance of the six apps on the three processors, we adapt the Roofline Performance model from highperformance computing (HPC) [58]. This simple visual model is not perfect, yet it offers insights into the causes of performance bottlenecks. The assumption behind the model is that applications don't fit in on-chip caches, so they are either computation-limited or memory bandwidth-limited. For HPC, the Y-axis is performance in floating-point operations per second, thus the peak computation rate forms the "flat" part of the roofline. The X-axis is operational intensity, measured as floating-point operations per DRAM byte accessed. Memory bandwidth is bytes per second, which turns into the "slanted" part of the roofline since $($ FLOPS/sec $) /($ FLOPS/Byte $)=$ Bytes/sec. Without sufficient operational intensity, a program is memory bandwidth-bound and lives under the slanted part of the roofline.

The gap between the actual operations per second of an application and the ceiling directly above it shows the potential benefit of further performance tuning while leaving operational intensity untouched; of course, optimizations that increase operational intensity (such as cache blocking) may yield even greater benefit.

To use the Roofline model for the TPU, when NN applications are quantized, we first replace floating-point operations with integer operations. As weights do not normally fit in on-chip memory for NN applications, the second change is to redefine operational intensity to be integer operations per byte of weights read (see the tenth column of Table 1).

Figure 5 shows the Roofline model for a single TPU die on $\log -\log$ scales. The TPU has a long "slanted" part of its roofline, where operational intensity means that performance is limited by memory bandwidth rather than by peak compute. Five of the six applications are happily bumping their heads against the ceiling: the MLPs and LSTMs are memory bound, and CNNs are computation bound. CNN1, despite a high operational intensity, is running at only 14.1 TOPS while CNN0 runs at 86 TOPS.

Table 3 explains what happened with $\mathrm{CNN} 1$, based on the performance counters that give us partial visibility into TPU operation. The TPU spends less than half of its cycles performing matrix operations for CNN1 (column 7, row 1). On each of those active cycles, only about half of the 65,536 MACs hold useful weights because some layers in $\mathrm{CNN} 1$ have shallow feature depths. About $35 \%$ of cycles are spent waiting for weights to load from memory into the matrix unit, which occurs during the 4 fully connected layers that run at an operational intensity of just 32 (see the last fallacy in Section 8). This leaves roughly $19 \%$ of cycles not explained by the matrix-related counters. Because of overlapped execution on the TPU, we do not have exact accounting for those cycles, but we can see that $23 \%$ of cycles have stalls for RAW dependences in the pipeline, and $1 \%$ are spent stalled for input over the PCIe bus.

Figures 6 and 7 show rooflines for a single Haswell die and for a single $\mathrm{K} 80$ die. The six NN applications are generally further below their ceilings than was the TPU in Figure 5. Response time is the reason. Many of these NN applications are parts of enduser-facing services. Researchers have demonstrated that small increases in response time cause customers to use a service less [51]. Hence, while training may not have hard response time deadlines, inference usually does. That is, inference prefers latency over throughput.

For example, the 99th-percentile response time limit for MLP0 was $7 \mathrm{~ms}$, which was required by the application developer. (The inferences per second and $7 \mathrm{~ms}$ latency include the server host time as well as the accelerator time.) Table 4 shows that Haswell and the K80 run at just $42 \%$ and $37 \%$, respectively, of the highest throughput achievable for MLP0 if the response time limit was relaxed. These bounds affect the TPU as well, but at $80 \%$ it is operating much closer to its highest MLP0 throughput. As compared to CPUs and GPUs, the single-threaded

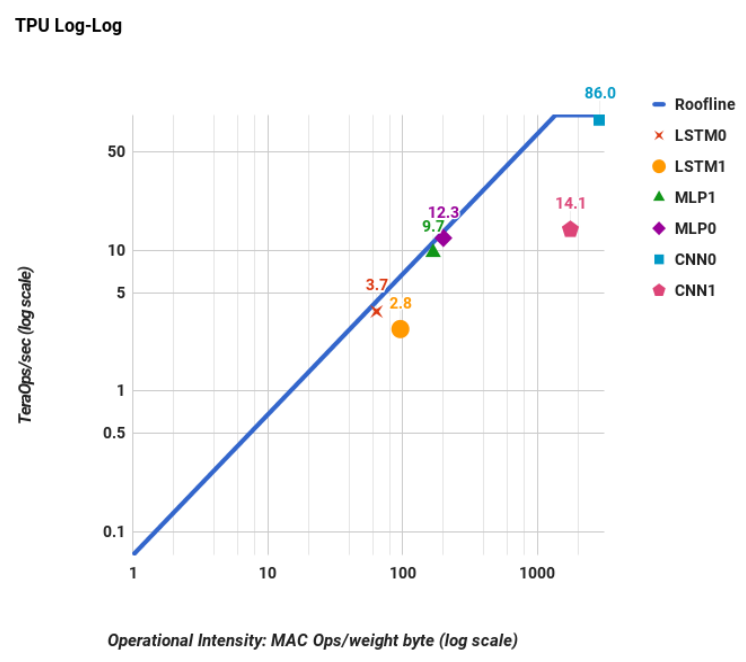

Figure 5. TPU (die) roofline. Its ridge point is far to the right at 1350 multiply-accumulate operations per byte of weight memory read. The 2 LSTMs and 2 MLPs are under the slanted part of the roofline, so they are bottlenecked by memory bandwidth on the TPU. The 2 CNNs are limited by the peak computation rate. 


\begin{tabular}{|c|c|c|c|c|c|c|c|c|}
\hline Application & $M L P O$ & $M L P 1$ & LSTMO & LSTM1 & CNNO & CNN1 & Mean & Row \\
\hline Array active cycles & $12.7 \%$ & $10.6 \%$ & $8.2 \%$ & $10.5 \%$ & $78.2 \%$ & $46.2 \%$ & $28 \%$ & 1 \\
\hline Useful MACs in $64 \mathrm{~K}$ matrix (\% peak) & $12.5 \%$ & $9.4 \%$ & $8.2 \%$ & $6.3 \%$ & $78.2 \%$ & $22.5 \%$ & $23 \%$ & 2 \\
\hline Unused MACs & $0.3 \%$ & $1.2 \%$ & $0.0 \%$ & $4.2 \%$ & $0.0 \%$ & $23.7 \%$ & $5 \%$ & 3 \\
\hline Weight stall cycles & $53.9 \%$ & $44.2 \%$ & $58.1 \%$ & $62.1 \%$ & $0.0 \%$ & $28.1 \%$ & $43 \%$ & 4 \\
\hline Weight shift cycles & $15.9 \%$ & $13.4 \%$ & $15.8 \%$ & $17.1 \%$ & $0.0 \%$ & $7.0 \%$ & $12 \%$ & 5 \\
\hline Non-matrix cycles & $17.5 \%$ & $31.9 \%$ & $17.9 \%$ & $10.3 \%$ & $21.8 \%$ & $18.7 \%$ & $20 \%$ & 6 \\
\hline RAW stalls & $3.3 \%$ & $8.4 \%$ & $14.6 \%$ & $10.6 \%$ & $3.5 \%$ & $22.8 \%$ & $11 \%$ & 7 \\
\hline Input data stalls & $6.1 \%$ & $8.8 \%$ & $5.1 \%$ & $2.4 \%$ & $3.4 \%$ & $0.6 \%$ & $4 \%$ & 8 \\
\hline TeraOps/sec (92 Peak) & 12.3 & 9.7 & 3.7 & 2.8 & 86.0 & 14.1 & 21.4 & 9 \\
\hline
\end{tabular}

Table 3. Factors limiting TPU performance of the NN workload based on hardware performance counters. Rows $1,4,5$, and 6 total $100 \%$ and are based on measurements of activity of the matrix unit. Rows 2 and 3 further break down the fraction of $64 \mathrm{~K}$ weights in the matrix unit that hold useful weights on active cycles. Our counters cannot exactly explain the time when the matrix unit is idle in row 6; rows 7 and 8 show counters for two possible reasons, including RAW pipeline hazards and PCIe input stalls. Row 9 (TOPS) is based on measurements of production code while the other rows are based on performance-counter measurements, so they are not perfectly consistent. Host server overhead is excluded here. CNN1 results are explained in the text.

TPU has none of the sophisticated microarchitectural features that consume transistors and energy to improve the average case but not the 99th-percentile case: no caches, branch prediction, out-oforder execution, multiprocessing, speculative prefetching, address coalescing, multithreading, context switching, and so forth. Minimalism is a virtue of domain-specific processors.

Table 3 shows TPU performance, but it doesn't account for host server time, which can be divided into running the host share of the application and talking to the TPU. Table 5 lists the second part, but the first part is hard. Queueing theory shows that long input queues raise throughput-by ensuring that the computer is never idle-but stretch response time. Thus, most applications keep their input queues empty. Alas, we can't measure when the TPU is idle since it is waiting for the CPU to do its portion of the application or because the CPU is also idle due to an empty input queue.

Table 6 gives the bottom line of relative inference performance per die including the host server overhead for the two accelerators versus the CPU. The next-to-last column shows the geometric mean of the relative performance for the six NN applications, which suggests the $\mathrm{K} 80$ die is $1.1 \mathrm{X}$ the speed of a Haswell die, that the TPU die is 14.5 times as fast, and thus the TPU die is 13.2 times as fast as the GPU die. Figure 8 shows their relative speeds visually.is

\begin{tabular}{|l|c|c|c|c|}
\hline Type & Batch & 99th\% Response & Inf/s (IPS) & \% Max IPS \\
\hline CPU & 16 & $7.2 \mathrm{~ms}$ & 5,482 & $42 \%$ \\
\hline CPU & 64 & $21.3 \mathrm{~ms}$ & 13,194 & $100 \%$ \\
\hline GPU & 16 & $6.7 \mathrm{~ms}$ & 13,461 & $37 \%$ \\
\hline GPU & 64 & $8.3 \mathrm{~ms}$ & 36,465 & $100 \%$ \\
\hline TPU & 200 & $7.0 \mathrm{~ms}$ & 225,000 & $80 \%$ \\
\hline TPU & 250 & $10.0 \mathrm{~ms}$ & 280,000 & $100 \%$ \\
\hline
\end{tabular}

Table 4. 99th\% response time and per die throughput (IPS) for MLP0 as batch size varies for MLP0. The longest allowable latency is $7 \mathrm{~ms}$. For the GPU and TPU, the maximum MLP0 throughput is limited by the host server overhead. Larger batch sizes increase throughput, but as the text explains, their longer response times exceed the limit, so CPUs and GPUs must use less-efficient, smaller batch sizes (16 vs. 200). They run $2.3 X-2.7 X$ slower than if response time was unbound, but the for the more deterministic TPU, the slowdown from the $99^{\text {th }} \%$ response-time limit is just $1.2 \mathrm{X}$.

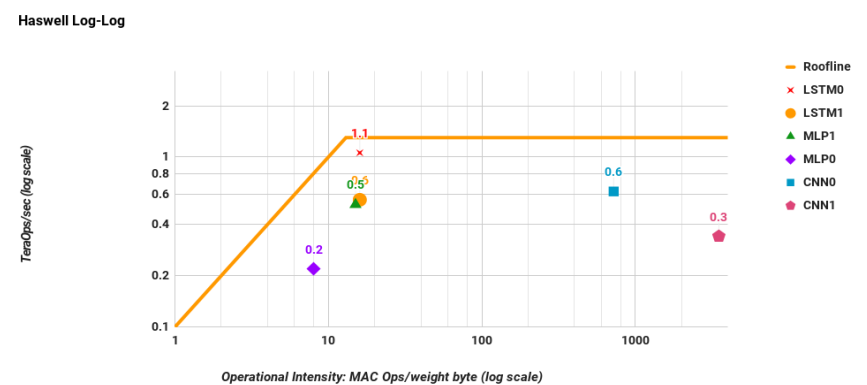

Figure 6. Intel Haswell CPU (die) roofline with its ridge point at 13 multiply-accumulate operations/byte, which is much further left than in Figure 5. LSTM0 and MLP1 are faster on Haswell than on the K80, but it is vice versa for the other DNNs. Response time limits DNN performance (Table 4).

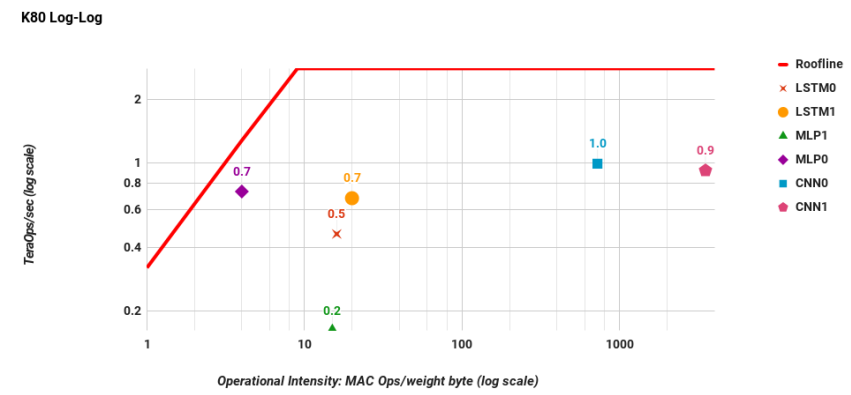

Figure 7. NVIDIA K80 GPU die Roofline. The higher memory bandwidth moves the ridge point to 9 operations per weight byte, which is further left than in Figure 6. The DNNs are far from their Roofline because of response time caps (Table 4).

\begin{tabular}{|c|c|c|c|c|c|}
\hline MLPO & MLP1 & LSTMO & LSTM1 & CNNO & CNN1 \\
\hline $21 \%$ & $76 \%$ & $11 \%$ & $20 \%$ & $51 \%$ & $14 \%$ \\
\hline
\end{tabular}

Table 5. Time for host CPU to interact with the TPU expressed as percent of TPU execution time (from TPU performance counters). This fraction is the time the CPU and TPU are communicating over the PCIe bus, not including the time the CPU is doing a portion of the application but not interacting with the TPU. As the text explains, it's hard for the TPU to measure if the CPU is idle or working on the application. 
Recall that architects use the geometric mean when they don't know the actual mix of programs that will be run [23]. For this study, however, we do know the mix (Table 1). The weighted mean in the last column of Table 6 using the actual mix increases the GPU to $1.9 \mathrm{X}$ and the TPU to $29.2 \mathrm{X}$, so the TPU die is now 15.3 times as fast as the GPU die.

\begin{tabular}{|l|r|r|r|r|r|r|r|r|}
\hline \multirow{2}{*}{ Type } & \multicolumn{2}{|c|}{ DNN } & \multicolumn{2}{c|}{ LSTM } & \multicolumn{2}{c|}{ CNN } & \multirow{2}{*}{ GM } & \multirow{2}{*}{ WM } \\
\cline { 2 - 8 } & \multicolumn{1}{|c|}{0} & \multicolumn{1}{c|}{1} & 0 & 1 & 0 & 1 & & \\
\hline GPU & 2.5 & 0.3 & 0.4 & 1.2 & 1.6 & 2.7 & 1.1 & 1.9 \\
\hline TPU & 41.0 & 18.5 & 3.5 & 1.2 & 40.3 & 71.0 & 14.5 & 29.2 \\
\hline Ratio & 16.7 & 60.0 & 8.0 & 1.0 & 25.4 & 26.3 & 13.2 & 15.3 \\
\hline
\end{tabular}

Table 6. K80 GPU die and TPU die performance relative to CPU for the NN workloads. GM and WM are geometric and weighted mean (using the mix from Table 1). Relative performance for the GPU and TPU includes host server overhead.

Log-Log Scale

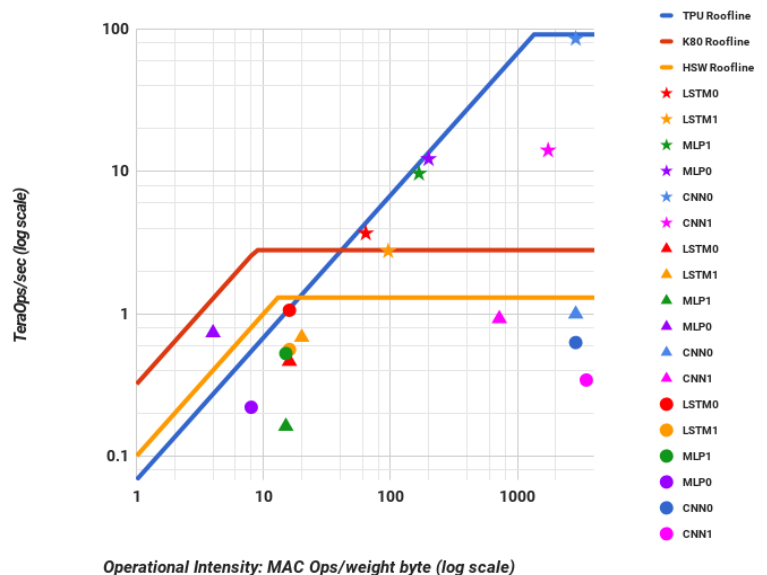

Figure 8. Figures 5-7 combined into a log-log graph. Stars are for the TPU, triangles are for the K80, and circles are for Haswell. All TPU stars are at or above the other two rooflines.

\section{COST-PERFORMANCE, TCO, AND PERFORMANCE/WATT}

When buying computers by the thousands, cost-performance trumps performance. The best cost metric in a datacenter is total cost of ownership (TCO). The actual price we pay for thousands of chips depends on negotiations between the companies involved. For business reasons, we can't publish such price information or data that might let them be deduced. However, power is correlated with TCO, and we can publish Watts per server, so we use performance/Watt as our proxy for performance/TCO in this paper. In this section, we compare whole servers rather than single dies, which Table 2 lists in the "Benchmarked Server" columns.

Figure 9 shows the geometric and weighted mean performance/Watt for the K80 GPU and TPU relative to the Haswell CPU. We present two different calculations of performance/Watt. The first ("total") includes the power consumed by the host CPU server when calculating performance/Watt for the GPU and TPU. The second ("incremental") subtracts the host CPU server power from the GPU and TPU beforehand.

For total-performance/Watt, the K80 server is $1.2 \mathrm{X}-2.1 \mathrm{X}$ Haswell. For incremental-performance/Watt, when Haswell server power is omitted, the K80 server is $1.7 \mathrm{X}-2.9 \mathrm{X}$. The TPU server has $17 \mathrm{X}-34 \mathrm{X}$ better total-performance/Watt than Haswell, which makes the TPU server $14 \mathrm{X}-16 \mathrm{X}$ the performance/Watt of the K80 server. The relative incremental-performance/Wattwhich was our company's justification for a custom ASIC - is $41 \mathrm{X}-83 \mathrm{X}$ for the TPU, which lifts the TPU to $25 \mathrm{X}-29 \mathrm{X}$ the performance/Watt of the GPU.

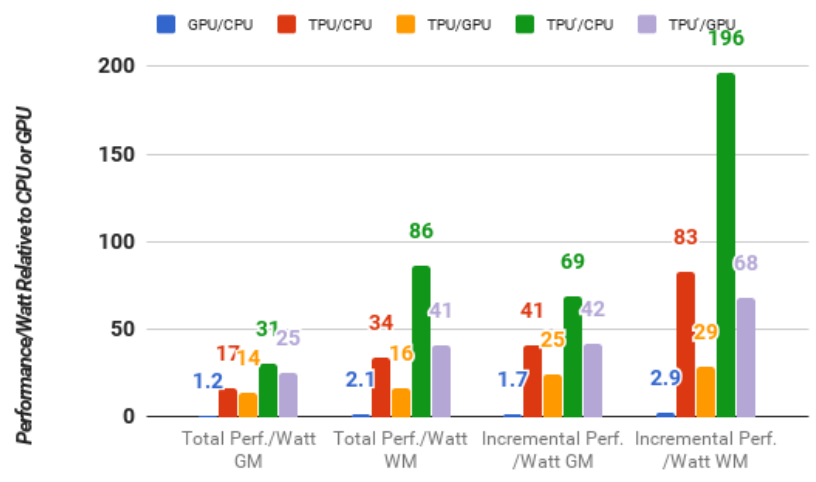

Figure 9. Relative performance/Watt (TDP) of GPU server (blue) and TPU server (red) to CPU server, and TPU server to GPU server (orange). TPU' is an improved TPU that uses GDDR5 memory (see Section 7). The green bar shows its ratio to the CPU server, and the lavender bar shows its relation to the GPU server. Total includes host server power, but incremental doesn't. GM and WM are the geometric and weighted means.

\section{ENERGY PROPORTIONALITY}

Thermal Design Power (TDP) affects the cost of provisioning power, as you must supply sufficient power and cooling when hardware is at full power. However, the cost of electricity is based upon the average consumed as the workload varies during the day. [6] found that servers are $100 \%$ busy less than $10 \%$ of the time and advocated energy proportionality: servers should consume power proportional to the amount of work performed. The estimate of power consumed in the prior section is based upon the fraction of the TDP that has been seen in our datacenters.

We measured performance and power of servers including CPUs, TPUs, and GPUs as the offered workload utilization varies from $0 \%$ to $100 \%$, collected in buckets of $10 \%$ delta of workload [32]. Figure 10 shows server power divided by the number of dies per server for the three chips by varying CNNO's workload.

We see that the TPU has the lowest power-40W per diebut it has poor energy proportionality: at $10 \%$ load, the TPU uses $88 \%$ of the power it uses at $100 \%$. (The short design schedule prevented inclusion of many energy-saving features.) Not surprisingly, Haswell is the best at energy proportionality of the group: it uses $56 \%$ of the power at $10 \%$ load as it does at $100 \%$. The $\mathrm{K} 80$ is closer to the CPU than the TPU, using $66 \%$ of the full load power at $10 \%$ workload. LSTM1, which is not computation bound, performs similarly: at $10 \%$ load the CPU uses $47 \%$ of full power, the GPU uses $78 \%$, and the TPU uses $94 \%$. 
What happens to the server power usage when running CNNO if it becomes a host to accelerators? When the GPU and TPU are at $100 \%$ load, the CPU server uses $52 \%$ of full power for the GPU and $69 \%$ for the TPU. (The CPU does more work for the TPU because it is running so much faster than the GPU.) Consequently, the Haswell server plus four TPUs use $<20 \%$ additional power but run CNNO 80 times faster than the Haswell server alone (4 TPUs vs. 2 CPUs).

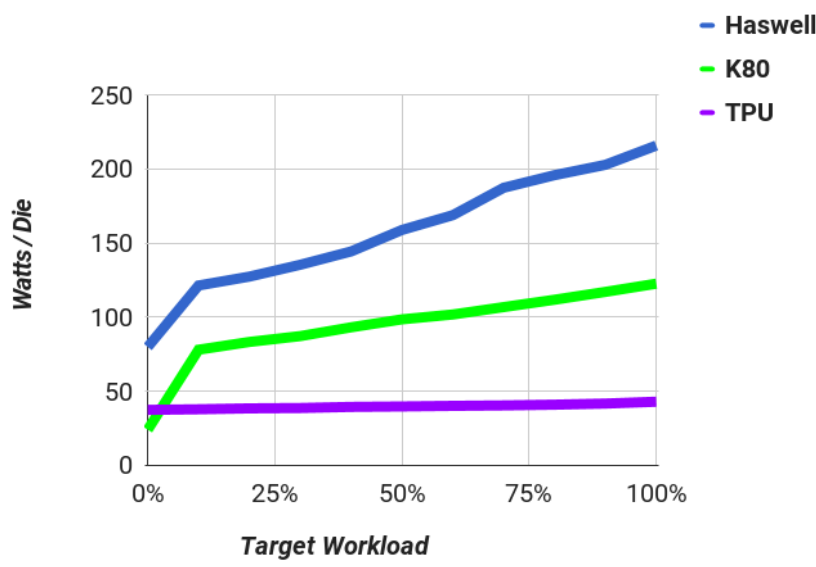

Figure 10. Watts/die for CNN0 as utilization varies from $0 \%$ to $100 \%$. A server has 2 CPUs and 8 GPUs or 4 TPUs, so we normalize power by dividing by 2,8 , and 4 , respectively.

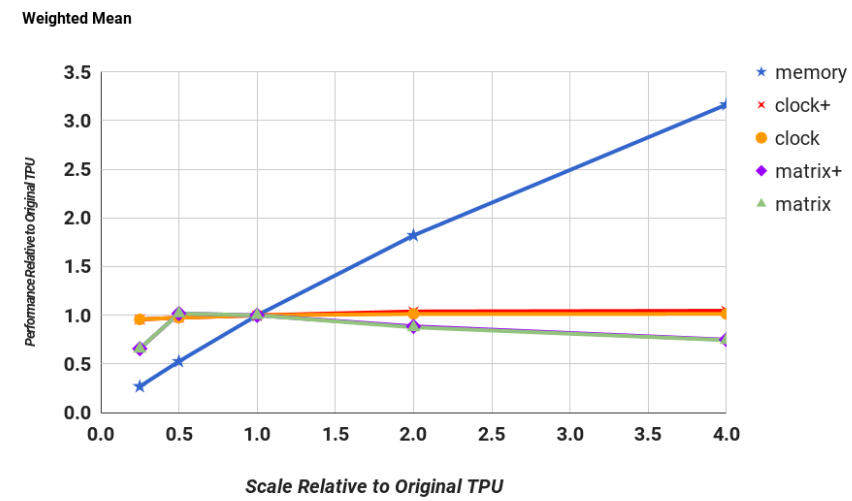

Figure 11. Weighted mean TPU performance as metrics scale from $0.25 x$ to $4 x$ : memory bandwidth (memory), clock rate + accumulators (clock +$)$, clock rate (clock), matrix unit

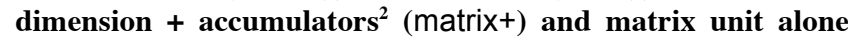
(matrix).

\begin{tabular}{|c|c|c|c|c|c|}
\hline MLP0 & MLP1 & LSTMO & LSTM1 & CNNO & CNN1 \\
\hline $6.8 \%$ & $10.9 \%$ & $7.7 \%$ & $5.4 \%$ & $8.2 \%$ & $11.2 \%$ \\
\hline
\end{tabular}

Table 7. Difference in clock cycles between the TPU hardware performance counters and the TPU performance model. The average delta is $8 \%$.

\begin{tabular}{|c|c|c|c|c|c|}
\hline$M L P 0$ & MLP1 & LSTMO & LSTM1 & CNNO & CNN1 \\
\hline 11.0 & 2.3 & 4.8 & 4.5 & 1.5 & 13.9 \\
\hline
\end{tabular}

Table 8. Maximum MiB of the $24 \mathrm{MiB}$ Unified Buffer used per NN application. A $14 \mathrm{MiB}$ Unified Buffer is sufficient for them now, due to improvements in the new software allocator.

\section{EVALUATION OF ALTERNATIVE TPU DESIGNS}

Like an FPU, the TPU coprocessor is relatively easy to evaluate, so we created a performance model for our six applications. Table 7 shows the differences between the model results and the hardware performance counters, which average below $10 \%$. We then modeled performance as we varied the memory bandwidth, the clock rate and number of accumulators, and the matrix multiply unit size.

Figure 11 shows the mean performance sensitivity of TPU die as we scale these parameters over the range for $0.25 \mathrm{x}$ to $4 \mathrm{x}$. It plots weighted means, but the geometric means look similar. In addition to evaluating the impact of only raising clock rates (clock in Figure 11), we also plot a design (clock+) where the clock rate is increased and the number of accumulators is correspondingly scaled so the compiler can keep more memory references in flight. Likewise, we plot matrix unit expansion if we increase the number of accumulators with the square of the rise in one dimension (matrix + ), since the number of multipliers in the matrix grows in both dimensions, as well as just increasing the matrix unit alone (matrix).

First, increasing memory bandwidth (memory) has the biggest impact: performance improves $3 \mathrm{X}$ on average when memory increases $4 \mathrm{X}$. Second, clock rate has little benefit on average with or without more accumulators. The reason is the MLPs and LSTMs are memory bound but only the CNNs are compute bound. While hard to see in Figure 11, since it shows only the weighted mean of all six DNNs, increasing the clock rate by $4 \mathrm{X}$ has almost no impact on MLPs and LSTMs but improves performance of CNNs by about $2 \mathrm{X}$. Third, the average performance in Figure 11 slightly degrades when the matrix unit expands from $256 \times 256$ to $512 \times 512$ for all apps, whether or not they get more accumulators. The issue is analogous to internal fragmentation of large pages, only worse since it's in two dimensions. Consider the $600 \times 600$ matrix used in LSTM1. With a $256 \times 256$ matrix unit, it takes 9 steps to tile $600 \times 600$, for a total of 18 us of time. The larger $512 \times 512$ unit requires only four steps, but each step takes four times longer, for 32 us of time. Our CISC instructions are long, so decode is insignificant and does not hide the overhead of loading from the DRAM.

Table 8 shows the utilization of the $24 \mathrm{MiB}$ Unified Buffer, which was initially sized to allow MLPs to run at batch sizes up to 2048. We recently improved the storage allocator for the Unified Buffer, which reduces the memory needed for the largest of the six applications to $14 \mathrm{MiB}$. For the first 18 months of deployment, the TPU used its full capacity while the new allocator was being developed. Now the extra capacity adds margin for adopting bigger models.

We next used the performance model to evaluate a hypothetical TPU die (TPU') that could be designed in the same process technology if we had more than 15 months. More aggressive logic synthesis and block design might have increased the clock rate by $50 \%$. Designing an interface circuit for GDDR5 memory, as in the $\mathrm{K} 80$, would improve Weight Memory bandwidth by more than a factor of five, shifting its roofline ridge point from 1350 to 250 . As Figure 11 shows, increasing clock rate to $1050 \mathrm{MHz}$ but not helping memory makes little change. If we left the clock at $700 \mathrm{MHz}$ but used GDDR5 for Weight Memory, the geometric mean increase jumps to 2.6 and the weighted mean 
to 3.9. Doing both raises the geometric mean (2.9) but not the weighted mean, so TPU' just has faster memory.

Figure 11 does not include host server time. We used Table 5 to calculate time for the host server interaction overhead for the TPU. Adding that same extra time drops TPU' means from 2.6 to 1.9 and 3.9 to 3.2. This change is both optimistic, since it doesn't include CPU time to run its share of the app, and pessimistic, as we likely would aggressively tune the host code given a $3 \mathrm{X}$ faster TPU'.

Replacing just the DDR3 Weight Memory with the K80equivalent GDDR5 memory requires doubling the number of memory channels to four. This improvement would expand die size by about $10 \%$. However, higher memory bandwidth reduces pressure on the Unified Buffer, so reducing the Unified Buffer to $14 \mathrm{MiB}$ could gain back $10 \%$ in area. GDDR5 would also increase the TPU system power budget from 861 Watts to about 900 Watts, as there are 4 TPUs per server.

Figure 9 above shows the relative total-performance /Watt/die of TPU' leaps to $31 \mathrm{X}-86 \mathrm{X}$ over Haswell and $25 \mathrm{X}-$ $41 \mathrm{X}$ over the K80. The incremental metric soars to $69 \mathrm{X}-196 \mathrm{X}$ over Haswell and 42X - 68X over the K80.

\section{DISCUSSION}

This section follows the fallacy and pitfall with rebuttal style of [23].

- Fallacy: NN inference applications in datacenters value throughput as much as response time.

We were surprised that our developers had strong response-time demands, as some suggested in 2014 that batch sizes would be large enough for the TPU to reach peak performance or that latency requirements would not be as tight. One driving application was off-line image processing, and the intuition was that if interactive services also wanted TPUs, most of them would just accumulate larger batches. Even the developers of one application in 2014 that cared about response time (LSTM1) said the limit was $10 \mathrm{~ms}$ in 2014 , but shrank it to $7 \mathrm{~ms}$ when they actually ported it to the TPU. The unexpected desire for TPUs by many such services combined with the impact on and preference for low response time changed the equation, with application writers often opting for reduced latency over waiting for bigger batches to accumulate. Fortunately, the TPU has a simple and repeatable execution model to help meet the response-time targets of interactive services and such high peak throughput that even small batch sizes result in higher performance than contemporary CPUs and GPUs.

- Fallacy: The K80 GPU architecture is a good match to NN inference.

GPUs have traditionally been seen as high-throughput architectures that rely on high-bandwidth DRAM and thousands of threads to achieve their goals. This perspective helps explain why the K80 is only a little faster at inference than Haswell and much slower than the TPU. Successors to the K80 will surely include optimizations to improve peak inference performance, but given their throughput-oriented architectural approach, it may be more challenging for GPUs to meet the strict latency limits. And as Section 7 shows, there is plenty of headroom to improve the TPU, so it's not an easy target.

- Pitfall: Architects neglected important NN tasks. We are pleased by the attention that the architecture community is paying to NN: $15 \%$ of the papers at ISCA 2016 were on hardware accelerators for $\mathrm{NN}[2,11,13,21,29,34,35,45,52]$ ! Alas, all nine papers looked at CNNs, and only two mentioned other NNs. CNNs are more complex than MLPs and are prominent in NN competitions [50], which might explain their allure, but they are only about $5 \%$ of our datacenter NN workload. While CNNs may be common in edge devices, the volume of convolutional models hasn't yet caught up with MLPs and LSTMs in the datacenter. We hope that architects try to accelerate MLPs and LSTMs with at least as much gusto.

- Pitfall: For NN hardware, Inferences Per Second (IPS) is an inaccurate summary performance metric.

Our results show that IPS is a poor overall performance summary for NN hardware, as it's simply the inverse of the complexity of the typical inference in the application (e.g., the number, size, and type of NN layers). For example, the TPU runs the 4-layer MLP1 at 360,000 IPS but the 89-layer CNN1 at only 4,700 IPS, so TPU IPS vary by $75 \mathrm{X}$ ! Thus, using IPS as the single-speed summary is even more misleading for NN accelerators than MIPS or FLOPS are for regular processors [23], so IPS should be even more disparaged. To compare $\mathrm{NN}$ machines better, we need a benchmark suite written at a high-level to port it to the wide variety of NN architectures. Fathom is a promising new attempt at such a benchmark suite [3].

- Fallacy: The K80 GPU results would be much better if Boost mode were enabled.

We didn't use K80 Boost mode but measured its impact on LSTM1. Boost mode increased the clock rate by a factor of up to 1.6 -from 560 to $875 \mathrm{MHz}$ - which increased performance by $1.4 \mathrm{X}$, but it also raised power by $1.3 \mathrm{X}$. The net gain in performance/Watt is $1.1 \mathrm{X}$, and thus Boost mode would have a minor impact on LSTM1.

- Fallacy: CPU and GPU results would be similar to the $T P U$ if we used them more efficiently or compared to newer versions.

We originally had 8-bit results for just one DNN on the CPU, due to the significant work to use AVX2 integer support efficiently. The benefit was $\sim 3.5 \mathrm{X}$. It was less confusing (and less space) to present all CPU results in floating point, rather than having one exception, with its own roofline. If all DNNs had similar speedup, performance/Watt ratio would drop from $41 \mathrm{X}-83 \mathrm{X}$ to $12 \mathrm{X}-$ $24 \mathrm{X}$. The new $16-\mathrm{nm}, 1.5 \mathrm{GHz}, 250 \mathrm{~W}$ P40 GPU can perform 47 Tera 8-bit ops/sec, but wasn't available in early 2015 , so isn't contemporary with our three platforms. We also can't know the fraction of P40 peak delivered within our rigid time bounds. (It also doesn't offer SECDED on internal memory, so we can't deploy it in our datacenters.) If we compared newer chips, Section 7 shows that we could triple performance of the $28-\mathrm{nm}, 0.7 \mathrm{GHz}$, 40W TPU just by using the K80's GDDR5 memory (at a cost of an additional 10W).

- Pitfall: Performance counters added as an afterthought for NN hardware.

The TPU has 106 performance counters, and we would like even more (see Table 3 ). The raison d'etre for $\mathrm{NN}$ accelerators is performance, and it is way too early in their evolution to have good intuition about what is going on.

- Fallacy: After two years of software tuning, the only path left to increase TPU performance is hardware upgrades.

The performance of CNN1 on the TPU could improve if developers and compiler writers did more work to match CNN1 to the TPU hardware. For example, developers could reorganize the applications to aggregate multiple short batches out of the 
convolution layers into a single, deeper batch (from 32 to 128) for the four fully connected layers. Such a single layer would improve utilization of the matrix unit (see Table 3 ). As CNN1 currently runs more than 70 times faster on the TPU than the CPU, the CNN1 developers are already very happy, so it's not clear whether or when such optimizations would be performed.

- Piffall: Being ignorant of architecture history when designing a domain-specific architecture.

Ideas that didn't fly for general-purpose computing may be ideal for domain-specific architectures. For the TPU, three important architectural features date back to the early 1980s: systolic arrays [31], decoupled-access/execute [54], and CISC instructions [41]. The first reduced the area and power of the large matrix multiply unit, the second fetches weights concurrently during operation of the matrix multiply unit, and the third better utilizes the limited bandwidth of the PCIe bus for delivering instructions. Historyaware architects could have a competitive edge.

\section{RELATED WORK}

Two survey articles document that custom NN ASICs go back at least 25 years [25, 4]. For example, CNAPS chips contained a 64 SIMD array of 16-bit by 8-bit multipliers, and several CNAPS chips could be connected together with a sequencer [19]. The Synapse-1 system was based upon a custom systolic multiplyaccumulate chip called the MA-16, which performed sixteen 16bit multiplies at a time [44]. The system concatenated several MA-16 chips together and had custom hardware to do activation functions.

Twenty-five SPERT-II workstations, accelerated by the T0 custom ASIC, were deployed starting in 1995 to do both NN training and inference for speech recognition [5]. The 40-Mhz T0 added vector instructions to the MIPS instruction set architecture. The eight-lane vector unit could produce up to sixteen 32-bit arithmetic results per clock cycle based on 8-bit and 16-bit inputs, making it 25 times faster at inference and 20 times faster at training than a SPARC-20 workstation. They found that 16 bits were insufficient for training, so they used two 16-bit words instead, which doubled training time. To overcome that drawback, they introduced "bunches" (batches) of 32 to 1000 data sets to reduce time spent updating weights, which made it faster than training with one word but no batches.

The more recent DianNao family of NN architectures minimizes memory accesses both on the chip and to external DRAM by having efficient architectural support for the memory access patterns that appear in NN applications [28, 11]. All use 16-bit integer operations and all designs synthesized down to layout, but no chips were fabricated. The original DianNao uses an array of 64 16-bit integer multiply-accumulate units with 44 $\mathrm{KB}$ of on-chip memory and is estimated to be $3 \mathrm{~mm}^{2}(65 \mathrm{~nm})$, to run at $1 \mathrm{GHz}$, and to consume $0.5 \mathrm{~W}$. Most of this energy went to DRAM accesses for weights, so one successor DaDianNao ("big computer") includes eDRAM to keep $36 \mathrm{MiB}$ of weights on chip. The goal was to have enough memory in a multichip system to avoid external DRAM accesses. The follow-on PuDianNao ("general computer") is aimed more at support vector machines. Another offshoot is ShiDianNao ("vision computer") aimed at CNNs, which avoids DRAM accesses by connecting the accelerator directly to the sensor.

The Convolution Engine is also focused on CNNs for image processing [43]. This design deploys 64 10-bit multiplyaccumulator units and customizes a Tensilica processor estimated to run at $800 \mathrm{MHz}$ in $45 \mathrm{~nm}$. It is projected to be $8 \mathrm{X}$ to $15 \mathrm{X}$ more energy-area efficient than an SIMD processor, and within $2 X$ to $3 \mathrm{X}$ of custom hardware designed just for a specific kernel.

The Fathom benchmark paper seemingly reports results contradictory to ours, with the GPU running inference much faster than the CPU [3]. However, their CPU and GPU are not serverclass, the CPU has only four cores, the applications do not use the CPU's AVX instructions, and there is no response-time cutoff [8].

Catapult [42] is the most widely deployed example of using reconfigurability to support DNNs, which many have proposed. They chose FPGAs over GPUs to reduce power as well as the risk that latency-sensitive applications wouldn't map well to GPUs. FPGAs can also be re-purposed, such as for search, compression, and network interface cards [Put15]. The TPU project actually began with FPGAs, but we abandoned them when we saw that the FPGAs of that time were not competitive in performance compared to the GPUs of that time, and the TPU could be much lower power than GPUs while being as fast or faster, potentially making it much better than FPGAs and GPUs.

Although first published in 2014, Catapult is a TPU contemporary since it deployed 28-nm Stratix V FPGAs into datacenters concurrently with the TPU in 2015. Catapult has a 200 MHz clock, 3,926 18-bit MACs, $5 \mathrm{MiB}$ of on-chip memory, 11 GB/s memory bandwidth, and uses 25 Watts. The TPU has a 700 MHz clock, 65,536 8-bit MACs, $28 \mathrm{MiB}, 34 \mathrm{~GB} / \mathrm{s}$, and typically uses 40 Watts. A revised version of Catapult was deployed at larger scale in 2016 [9].

Catapult V1 runs CNNs-using a systolic matrix multiplier-2.3X as fast as a $2.1 \mathrm{GHz}, 16$-core, dual-socket server [39]. Using the next generation of FPGAs (14-nm Arria 10) of Catapult V2, performance might go up to $7 \mathrm{X}$, and perhaps even $17 \mathrm{X}$ with more careful floor-planning [40]. Although it's apples versus oranges, a current TPU die runs its CNNs $40 \mathrm{X}$ to $70 \mathrm{X}$ versus a somewhat faster server (Tables 2 and 6). Perhaps the biggest difference is that to get the best performance the user must write long programs in the low-level hardware-design-language Verilog [36] versus writing short programs using the high-level TensorFlow framework. That is, reprogrammability comes from software for the TPU rather than from firmware for the FPGA.

\section{CONCLUSION}

Despite living on an $\mathrm{I} / \mathrm{O}$ bus and having relatively low memory bandwidth that limits utilization of the TPU - four of the six NN applications are memory-bound-a small fraction of a big number can nonetheless be relatively large, as the Roofline performance model demonstrates. This result suggests a "Cornucopia Corollary" to Amdahl's Law: low utilization of a huge, cheap resource can still deliver high, cost-effective performance.

The TPU leverages the order-of-magnitude reduction in energy and area of 8-bit integer systolic matrix multipliers over 32-bit floating-point datapaths of a K80 GPU to pack 25 times as many MACs (65,536 8-bit vs. 2,496 32-bit) and 3.5 times the onchip memory ( $28 \mathrm{MiB}$ vs. $8 \mathrm{MiB}$ ) while using less than half the power of the K80 in a relatively small die. This larger memory helps increase the operational intensity of applications to let them utilize the abundant MACs even more fully.

We found that despite a recent emphasis on CNNs in the architecture community, they constitute only about $5 \%$ of the representative NN workload for our datacenters, which suggests more attention should be paid to MLPs and LSTMs. Repeating history, it's similar to when many architects concentrated on 
floating-point performance when most mainstream workloads turned out to be dominated by integer operations.

We observed that inferences per second (IPS) is more a function of the NN than of the underlying hardware, and so IPS is an even worse single performance metric for NN processors than MIPS and MFLOPS are for CPUs and GPUs.

We also learned that inference applications have serious response-time bounds because they are often part of user facing applications, thus NN architectures need to perform well when coping with 99th-percentile latency deadlines. While the K80 may excel at training, on average it is just a little faster than Haswell at inference for our workload, perhaps because of its emphasis on throughput rather than latency; that conflicts with the strict response-time deadlines of our inference applications.

The TPU die leverages its advantage in MACs and on-chip memory to run short programs written using the domain-specific TensorFlow framework 15 times as fast as the K80 GPU die, resulting in a performance per Watt advantage of 29 times, which is correlated with performance per total cost of ownership. Compared to the Haswell CPU die, the corresponding ratios are 29 and 83. While future CPUs and GPUs will surely run inference faster, a redesigned TPU using circa 2015 GPU memory would go two to three times as fast and boost the performance/Watt advantage to nearly 70 over the K80 and 200 over Haswell.

In summary, the TPU succeeded because of the large-but not too large-matrix multiply unit; the substantial softwarecontrolled on-chip memory; the ability to run whole inference models to reduce dependence on its host CPU; a single-threaded, deterministic execution model that proved to be a good match to 99th-percentile response time limits; enough flexibility to match the NNs of 2017 as well as of 2013; the omission of generalpurpose features that enabled a small and low power die despite the larger datapath and memory; the use of 8-bit integers by the quantized applications; and that applications were written using TensorFlow, which made it easy to port them to the TPU at highperformance rather than them having to be rewritten to run well on the very different TPU hardware.

Order-of-magnitude differences between products are rare in computer architecture, which may lead to the TPU becoming an archetype for domain-specific architectures. We expect that many will build successors that will raise the bar even higher.

\section{ACKNOWLEDGEMENTS}

We thank the leadership of our company for recognizing the need for a TPU and for providing the resources to build, distribute, evaluate, and publish. Special thanks go to Luiz Barroso and James Laudon for helping start the project. It takes a village to design, verify, and implement the hardware and software of a system like a TPU and to manufacture, deploy, and use it at scale, which is why there are many authors. (All authors but Dave Patterson worked on the TPU; he joined in 2016.) The first four authors did the bulk of the evaluation in this paper, which is why they are in front, with the rest in alphabetical order. Norm Jouppi was also the senior architect of the whole project; the reward for his yeoman's work is being the lead author.

\section{REFERENCES}

[1] Abadi, M., Agarwal, A., Barham, P., Brevdo, E., Chen, Z., Citro, C., Corrado, G.S., Davis, A., Dean, J., Devin, M. Ghemawat, S., et al. 2016. TensorFlow: Large-scale machine learning on heterogeneous distributed systems. arXiv:1603.04467.

[2] Albericio, J., Judd, P., Hetherington, T., Aamodt, T., Jerger, N.E. and Moshovos, A., 2016 Cnvlutin: Ineffectual-Neuron-Free Deep Neural Network Computing. Proc. Int'l Symp. on Computer Architecture.

[3] Adolf, R., Rama, S., Reagen, B., Wei, G.Y. and Brooks, D., 2016, September. Fathom: reference workloads for modern deep learning methods. IEEE Int'l Symp. on Workload Characterization (IISWC).

[4] Asanović, K. 2002. Programmable Neurocomputing, in The Handbook of Brain Theory and Neural Networks: Second Edition, M. A. Arbib (Ed.), MIT Press, ISBN 0-262-01197-2, November 2002.

[5] Asanović, K. 1998. Asanović, K., Beck, Johnson, J., Wawrzynek, J., Kingsbury, B. and Morgan, N., November 1998. Training Neural Networks with Spert-II. Chapter 11 in Parallel Architectures for Artificial Networks: Paradigms and Implementations, $\mathrm{N}$. Sundararajan and P. Saratchandran (Eds.), IEEE Computer Society Press, ISBN 0-8186-8399-6. https://people.eecs.berkeley.edu/ $\sim$ krste/papers/annbook.pdf

[6] Barroso, L.A. and Hölzle, U., 2007. The case for energyproportional computing. IEEE Computer, vol. 40.

[7] Barr, J. September 29, 2016, New P2 Instance Type for Amazon EC2 - Up to 16 GPUs. https://aws.amazon.com/blogs /aws/new-p2instance-type-for-amazon-ec2-up-to-16-gpus/

[8] Brooks, D. November 4, 2016. Private communication.

[9] Caulfield, A.M., Chung, E.S., Putnam, A., Angepat, H., Fowers, J., Haselman, M., Heil, S., Humphrey, M., Kaur, P., Kim, J.Y. and Lo, D.2016. A Cloud-Scale Acceleration Architecture. MICRO-49 conference.

[10] Cavigelli, L., Gschwend, D., Mayer, C., Willi, S., Muheim, B. and Benini, L., 2015, May. Origami: A convolutional network accelerator. Proc. 25th edition on Great Lakes Symp. on VLSI.

[11] Chen, Y.H., Emer, J. and Sze, V., 2016. Eyeriss: A Spatial Architecture for Energy-Efficient Dataflow for Convolutional Neural Networks. Proc. Int'l Symp. on Computer Architecture.

[12] Chen, Y., Chen, T., Xu, Z., Sun, N., and Teman, O., 2016. DianNao Family: Energy-Efficient Hardware Accelerators for Machine Learning, Research Highlight, CACM, 59(11).

[13] Chi, P., Li, S., Qi, Z., Gu, P., Xu, C., Zhang, T., Zhao, J., Liu, Y., Wang, Y. and Xie, Y., 2016. PRIME: A Novel Processing-InMemory Architecture for Neural Network Computation in ReRAMbased Main Memory. Proc. Int'l Symp. on Computer Architecture.

[14] Clark, J. October 26, 2015, Google Turning Its Lucrative Web Search Over to AI Machines. Bloomberg Technology, http://www.bloomberg.com.

[15] Dally, W. February 9, 2016. High Performance Hardware for Machine Learning, Cadence ENN Summit.

[16] Dean, J. and Barroso, L.A., 2013. The tail at scale. CACM, 56(2).

[17] Dean, J. July 7, 2016 Large-Scale Deep Learning with TensorFlow for Building Intelligent Systems, ACM Webinar.

[18] Gupta, S., Agrawal, A., Gopalakrishnan, K., and Narayanan, P., 2015, July. Deep Learning with Limited Numerical Precision. ICML.

[19] Hammerstrom, D., 1990, June. A VLSI architecture for highperformance, low-cost, on-chip learning. 1990 IJCNN Int'l Joint Conference on Neural Networks.

[20] Han, S.; Pool, J.; Tran, J.; and Dally, W., 2015. Learning both weights and connections for efficient neural networks. In Advances in Neural Information Processing Systems.

[21] Han, S., Liu, X., Mao, H., Pu, J., Pedram, A., Horowitz, M.A. and Dally, W.J., 2016. EIE: efficient inference engine on compressed deep neural network. Proc. Int'l Symp. on Computer Architecture.

[22] He, K., Zhang, X., Ren, S. and Sun, J., 2016. Identity mappings in deep residual networks. Also in arXiv preprint arXiv:1603.05027. 
[23] Hennessy, J.L. and Patterson, D.A., 2018. Computer architecture: a quantitative approach, 6th edition, Elsevier.

[24] Hölzle, U. and Barroso, L., 2009. The datacenter as a computer. Morgan and Claypool.

[25] Ienne, P., Cornu, T. and Kuhn, G., 1996. Special-purpose digital hardware for neural networks: An architectural survey. Journal of VLSI signal processing systems for signal, image and video technology, 13(1).

[26] Intel, 2016, Intel ${ }^{\circledR} \quad$ Xeon ${ }^{\circledR}$ Processor E5-4669 v3, http://ark.intel.com/products/85766/Intel-Xeon-Processor-E5-4669v3-45M-Cache-2_10-GHz.

[27] Jouppi, N. May 18, 2016. Google supercharges machine learning tasks with TPU custom chip. https://cloudplatform.googleblog.com.

28] Keutzer, K., 2016. If I could only design one circuit...: technical perspective. CACM, 59(11).

[29] Kim, D., Kung, J.H., Chai, S., Yalamanchili, S. and Mukhopadhyay, S., 2016. Neurocube: A Programmable Digital Neuromorphic Architecture with High-Density 3D Memory. Proc. Int'l Symp. on Computer Architecture.

30] Krizhevsky, A., Sutskever, I. and Hinton, G., 2012. Imagenet classification with deep convolutional neural networks. Advances in neural information processing systems.

[31] Kung, H.T. and Leiserson, C.E., 1980. Algorithms for VLSI processor arrays. Introduction to VLSI systems.

[32] Lange, K.D., 2009. Identifying shades of green: The SPECpower benchmarks. IEEE Computer, 42(3).

[33] Larabel, M. March 10, 2016, Google Looks To Open Up StreamExecutor To Make GPGPU Programming Easier, Phoronix, https://www.phoronix.com/scan.php?page=news_item\&px=GoogleStreamExec-Parallel.

[34] LiKamWa, R., Hou, Y., Gao, J., Polansky, M. and Zhong, L., 2016. RedEye: Analog ConvNet Image Sensor Architecture for Continuous Mobile Vision. Proc. Int'l Symp. on Computer Architecture.

[35] Liu, S., Du, Z.D., Tao, J.H., Han, D., Luo, T., Xie, Y., Chen, Y. and Chen, T., 2016. Cambricon: An instruction set architecture for neural networks. Proc. Int'l Symp. on Computer Architecture.

[36] Metz, C. September 26, 2016, Microsoft Bets Its Future On A Reprogrammable Computer Chip, Wired Magazine, https://www.wired.com/2016/09/microsoft-bets-future-chipreprogram-fly/

[37] Nvidia, January 2015. Tesla K80 GPU Accelerator. Board Specification https://images.nvidia.com/ content/pdf/kepler/TeslaK80-BoardSpec-07317-001-v05.pdf.

[38] Nvidia, 2016. Tesla GPU Accelerators For Servers. http://www.nvidia.com/object/tesla-servers.html.

[39] Ovtcharov, K., Ruwase, O., Kim, J.Y., Fowers, J., Strauss, K. and Chung, E.S., February 2, 2015. Accelerating deep convolutional neural networks using specialized hardware. Microsoft Research Whitepaper. www.microsoft.com/en-us/research/publication/ accelerating-deep-convolutional-neural-networks-using-specializedhardware/

[40] Ovtcharov, K., Ruwase, O., Kim, J.Y., Fowers, J., Strauss, K. and Chung, E.S., 2015, August. Toward accelerating deep learning at scale using specialized hardware in the datacenter. 2015 IEEE Hot Chips 27 Symp.

[41] Patterson, D.A. and Ditzel, D.R., 1980. The case for the reduced instruction set computer. ACM SIGARCH Computer Architecture News, 8(6), pp. 25-33.

[42] Putnam, A., Caulfield, A.M., Chung, E.S., Chiou, D., Constantinides, K., Demme, J., Esmaeilzadeh, H., Fowers, J., Gopal, G.P., Gray, J., Haselman, M., Hauck, S., Heil, S., Hormati, A., Kim, J-Y., Lanka, S., Larus, J., Peterson, E., Pope, S., Smith, A., Thong, J., Xiao, P.Y., Burger, D. 2016. A Reconfigurable Fabric for Accelerating Large-Scale Datacenter Services. CACM, 59(11).
[43] Qadeer, W., Hameed, R., Shacham, O., Venkatesan, P., Kozyrakis, C. and Horowitz, M.A., 2013, June. Convolution engine: balancing efficiency \& flexibility in specialized computing. Proc. Int'l Symp. on Computer Architecture.

[44] Ramacher, U., Beichter, J., Raab, W., Anlauf, J., Bruels, N., Hachmann, U. and Wesseling, M., 1991. Design of a 1st Generation Neurocomputer. In VLSI Design of Neural Networks. Springer US

[45] Reagen, B., Whatmough, P., Adolf, R., Rama, S., Lee, H., Lee, S.K., Hernández-Lobato, J.M., Wei, G.Y. and Brooks, D., 2016. Minerva: Enabling low-power, highly-accurate deep neural network accelerators. Proc. Int'l Symp. on Computer Architecture.

[46] Ross, J., Jouppi, N., Phelps, A., Young, C., Norrie, T., Thorson, G., Luu, D., 2015. Neural Network Processor, Patent Application No. 62/164,931

[47] Ross, J., Phelps, A., 2015. Computing Convolutions Using a Neural Network Processor, Patent Application No. 62/164,902.

[48] Ross, J., 2015. Prefetching Weights for a Neural Network Processor, Patent Application No. 62/164,981.

[49] Ross, J., Thorson, G., 2015. Rotating Data for Neural Network Computations, Patent Application No. 62/164,908.

[50] Russakovsky, O., Deng, J., Su, H., Krause, J., Satheesh, S., Ma, S., Huang, Z., Karpathy, A., Khosla, A., Bernstein, M. and Berg, A.C., 2015. Imagenet large scale visual recognition challenge. Int'l Journal of Computer Vision, 115(3).

[51] Schurman, E. and Brutlag, J., 2009, June. The user and business impact of server delays, additional bytes, and HTTP chunking in web search. In Velocity Web Performance and Operations Conference.

[52] Shafiee, A., Nag, A., Muralimanohar, N., Balasubramonian, R., Strachan, J.P., Hu, M., Williams, R.S. and Srikumar, V., 2016. ISAAC: A Convolutional Neural Network Accelerator with In-Situ Analog Arithmetic in Crossbars. Proc. Int'l Symp. on Computer Architecture.

[53] Silver, D., Huang, A., Maddison, C.J., Guez, A., Sifre, L., Van Den Driessche, G., Schrittwieser, J., Antonoglou, I., Panneershelvam, V. Lanctot, M. and Dieleman, S., 2016. Mastering the game of Go with deep neural networks and tree search. Nature, 529(7587).

[54] Smith, J.E., 1982, April. Decoupled access/execute computer architectures. Proc. Int'l Symp. on Computer Architecture.

[55] Steinberg, D., 2015. Full-Chip Simulations, Keys to Success. Proc. Synopsys Users Group (SNUG) Silicon Valley 2015.

[56] Szegedy, C., Liu, W., Jia, Y., Sermanet, P., Reed, S., Anguelov, D., Erhan, D., Vanhoucke, V. and Rabinovich, A., 2015. Going deeper with convolutions. Proc. IEEE Conference on Computer Vision and Pattern Recognition.

[57] Thorson, G., Clark, C., Luu, D., 2015. Vector Computation Unit in a Neural Network Processor, Patent Application No. 62/165,022.

[58] Williams, S., Waterman, A. and Patterson, D., 2009. Roofline: an insightful visual performance model for multicore architectures. CACM, 52(4).

[59] Wu, Y., Schuster, M., Chen, Z., Le, Q., Norouzi, M., Macherey, W., Krikun, M., Cao, Y., Gao, Q., Macherey, K., Klingner, J., Shah, A., Johnson, M., Liu, X., Kaiser, Ł., Gouws, S., Kato, Y., Kudo, T., Kazawa, H., Stevens, K., Kurian, G., Patil, N., Wang, W., Young, C., Smith, J., Riesa, J., Rudnick, A., Vinyals, O., Corrado, G., Hughes, M., and Dean, J. September 26, 2016, Google's Neural Machine Translation System: Bridging the Gap between Human and Machine Translation, http://arxiv.org/abs/1609.08144.

[60] Young, C., 2015. Batch Processing in a Neural Network Processor, Patent Application No. 62/165,020.

[61] Zhang, C., Li, P., Sun, G., Guan, Y., Xiao, B. and Cong, J., 2015, February. Optimizing FPGA-based accelerator design for deep convolutional neural networks. Proceedings of the 2015 ACM/SIGDA International Symposium on Field-Programmable Gate Arrays. 